\title{
RADIX CONVERSION FOR IEEE754-2008 MIXED RADIX FlOATING-PoINT ARITHMETIC
}

\author{
Olga Kupriianova \\ Christoph Lauter \\ Jean-Michel Muller \\ UPMC Paris 6 - LIP6 - PEQUAN team UPMC Paris 6 - LIP6 - PEQUAN team CNRS - ENS Lyon - Université de Lyon \\ 4, place Jussieu \\ 4 , place Jussieu \\ 46, allée d'Italie \\ 75252 Paris Cedex 05, France \\ 75252 Paris Cedex 05, France \\ 69364 Lyon Cedex 07, France \\ Email: olga.kupriianova@lip6.fr \\ Email: christoph.lauter@lip6.fr \\ Email: jean-michel.muller@ens-lyon.fr
}

\begin{abstract}
Conversion between binary and decimal floatingpoint representations is ubiquitous. Floating-point radix conversion means converting both the exponent and the mantissa. We develop an atomic operation for FP radix conversion with simple straight-line algorithm, suitable for hardware design. Exponent conversion is performed with a small multiplication and a lookup table. It yields the correct result without error. Mantissa conversion uses a few multiplications and a small lookup table that is shared amongst all types of conversions. The accuracy changes by adjusting the computing precision.
\end{abstract}

\section{INTRODUCTION}

Humans are used to operate decimals while almost all the hardware is binary. According to IEEE754-2008 norm [1] a floating point number is represented as $\beta^{E} \cdot m$, where $\beta^{p-1} \leq m \leq \beta^{p}-1 ; p$ is precision, $m \in \mathbb{N}$ is mantissa, $E \in \mathbb{Z}$ is exponent and $\beta$, the base or radix, is either two or ten. When the base $\beta=2$, we have binary floating point (FP) numbers, when $\beta=10$, the decimal one. However, most of hardware is binary, so the decimal mantissas are actually coded in binary. The formats for both radices differ by the length of stored numbers. Standartization of decimal FP arithmetic brings new challenges, e.g. supporting decimal transcendental functions with essentially binary hardware [2]. In [2] in order to evaluate decimal transcendental function the format conversion is used twice. The IEEE standard requires [1] the implementation of all the operations for different formats, but only for the operands of the same radix. The format does not require any mixed radix operations, i.e. one of the operands is binary, the other is decimal. Mixed radix arithmetic is currently being developed, although there are already some approaches published [3], [4].

Floating point radix conversion (from binary to decimal and vice versa) is a widespread operation, the simplest examples are the scanf and print $f$ functions. It could also exist as an operation for financial applications or as a "precomputing step" for mixed radix operations. The radix conversion is used in number conversion operations, and implicitly in scan $f$ and printf operations.

The current implementations of scanf and print $f$ are correct only for one rounding mode and allocate a lot of memory. In this paper we develop a unified atomic operation for the conversion, so all the computations can be done in integer with the precomputed memory consumption.
While radix conversion is a very common operation, it comes in different variants that are mostly coded in ad-hoc way in existing code. However, radix conversion always breaks down into to elementary steps: determining an exponent of the output radix and computing a mantissa in the output radix. Section II describes the 2-steps approach of the radix conversion, section III contains the algorithm for the exponent computation, section IV presents a novel approach of raising 5 to an integer power used in the second step of the radixconversion that computes the mantissa. Section V contains accuracy bounds for the algorithm of raising five to a huge power, section VI describes some implementation tricks and presents experimental results.

\section{Two-STEPS RAdiX CONVERsion Algorithm}

Conversion from a binary FP representation $2^{E} \cdot m$, where $E$ is the binary exponent and $m$ is the mantissa, to a decimal representation $10^{F} \cdot n$, requires two steps: determination of the decimal exponent $F$ and computation of the mantissa $n$. The conversion back to binary is pretty similar except of an extra step that will be explained later. Here and after consider the normalized mantissas $n$ and $m: 10^{p_{10}-1} \leq n \leq 10^{p_{10}}-1$ and $2^{p_{2}-1} \leq m \leq 2^{p_{2}}-1$, where $p_{10}$ and $p_{2}$ are the decimal and binary precisions respectively. The exponents $F$ and $E$ are bounded by some values depending on the IEEE754-2008 format.

In order to enclose the converted decimal mantissa $n$ into one decade, for a certain output precision $p_{10}$, the decimal exponent $F$ has to be computed [5] as follows:

$$
F=\left\lfloor\log _{10}\left(2^{E} \cdot m\right)\right\rfloor-p_{10}+1 .
$$

The most difficult thing here is the evaluation of the logarithm: as the function is transcendental, the result is always an approximation and function call is extremely expensive. Present algorithm computes the exponent (1) for a new-radix floating-point number only with a multiplication, binary shift, a precomputed constant and a lookup table (see section III).

Once $F$ is determined, the mantissa $n$ is given as

$$
n=* p_{10}\left(\frac{2^{E} \cdot m}{10^{F}}\right),
$$

where $*_{p_{10}}$ corresponds to the current rounding mode (to the nearest, rounding down, or rounding up [1]). The conversions are always done with some error $\varepsilon$, so the following relation is 
fulfilled: $10^{F} \cdot n=2^{E} \cdot m \cdot(1+\varepsilon)$. In order to design a unique algorithm for all the rounding modes it is useful to compute $n^{*}$, such that $10^{F} \cdot n^{*}=2^{E} \cdot m$. Thus, we get the following expression for the decimal mantissa:

$$
n^{*}=2^{E-F} 5^{-F} m
$$

As $2^{E-F}$ is a simple binary shift and the multiplication by $m$ is small, the binary-to-decimal mantissa conversion reduces to compute the leading bits of $5^{-F}$.

The proposed ideas apply with minor changes to decimalto-binary conversion: the base of the logarithm is 2 on the exponent computation step and one additional step is needed; for the mantissa computation the power $5^{F}$ is required instead of $5^{-F}$.

\section{LOOP-LESS EXPONENT DETERMINATION}

The current implementations of the logarithm function are expensive and produce approximated values. However, some earlier conversion approaches computed this approximation [6] by Taylor series or using iterations [7], [8]. Here the exponent for the both conversions is computed exactly neither with libm function call nor any polynomial approximation.

After performing one transformation step, (1) can be rewritten as following:

$$
F=\left\lfloor E \log _{10}(2)+\left\lfloor\log _{10}(m)\right\rfloor+\left\{\log _{10}(m)\right\}\right\rfloor-p_{10}+1,
$$

where $\{x\}=x-\lfloor x\rfloor$, the fractional part of the number $x$.

As the binary mantissa $m$ is normalized in one binade $2^{p_{2}-1} \leq m<2^{p_{2}}$, we can assume that it lies entirely in one decade. If it is not the case, we can always scale it a little bit. The inclusion in one decade means that $\left\lfloor\log _{10}(m)\right\rfloor$ stays the same on the whole interval. So, for the given format one can precompute and store this value as a constant. Thus, it is possible to take the integer number $\left\lfloor\log _{10}(m)\right\rfloor$ out of the floor operation in the previous equation. After representing the first summand as a sum of it's integer and fractional parts, we have the following expression under the floor operation:

$$
\left\lfloor\left\lfloor E \log _{10}(2)\right\rfloor+\left\{E \log _{10}(2)\right\}+\left\{\log _{10}(m)\right\}\right\rfloor .
$$

Here we add two fractional parts to an integer. We add something that is strictly less than two, so under the floor operation we have either an integer plus some small fraction that will be thrown away, or an integer plus one plus small fraction. Thus, we can take the fractional parts out of the floor brackets adding a correction $\gamma$ :

$$
\left\lfloor E \log _{10}(2)\right\rfloor+\gamma, \gamma \in\{0,1\} .
$$

This correction $\gamma$ equals to 1 when the sum of two fractional parts from the previous expression exceeds 1 , or mathematically:

$$
E \log _{10}(2)-\left\lfloor E \log _{10}(2)\right\rfloor+\log _{10}(m)-\left\lfloor\log _{10}(m)\right\rfloor \geq 1 .
$$

Due to the logarithm function the expression on the left is strictly monotonous (increasing). This means that we need only one threshold value $m^{*}(E)$, such that $\forall m \geq m^{*}(E)$ the correction $\gamma=1$. As we know the range for the exponents $E$ beforehand, we can store the critical values $m^{*}(E)=$

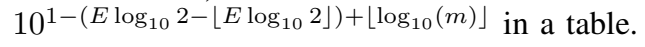

There is a technique proposed in [9] to compute $\left\lfloor E \log _{10}(2)\right\rfloor$ with a multiplication, binary shift and the use of a precomputed constant. So, finally the value of the decimal exponent can be obtained as

$$
F=\left\lfloor E\left\lfloor\log _{10}(2) \cdot 2^{\lambda}\right\rfloor \cdot 2^{-\lambda}\right\rfloor+\left\lfloor\log _{10}(m)\right\rfloor-p_{10}+1+\gamma
$$

The algorithm pseudocode is provided below.

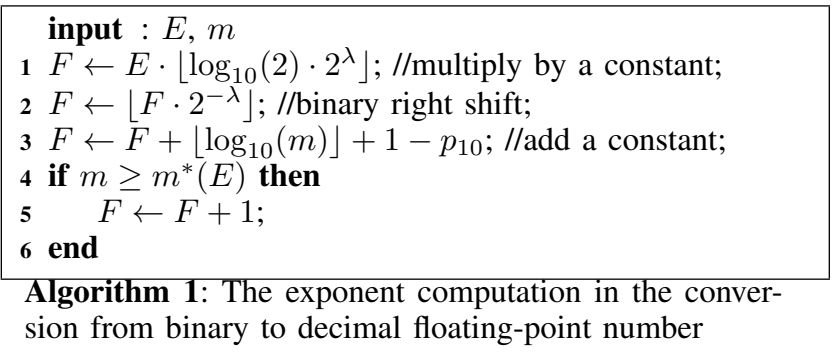

The decimal-to-binary conversion algorithm is the same with a small additional remark. We want to convert decimal FP numbers to binary, so the input mantissas are in the range $10^{p_{10}-1} \leq n<10^{p_{10}}$. As we mentioned, on this step the base of the logarithm is 2 , and the problem here is that $\left\lfloor\log _{2}(10)\right\rfloor=3$, so it seems that we need three tables, but once we represent the decimal mantissa $n$ as a binary FP number $n=2^{\hat{E}} \hat{m}$ in some precision $\kappa$, it suffices just one table. For all the possible values $\hat{m}$ the following holds $\left\lfloor\log _{2}(\hat{m})\right\rfloor=\kappa-1$. This mantissa representation can be made exact: we'll have to shift the decimal $n$ to the left. Thus, the precision of this new number is $\kappa=\left\lceil\log _{2}\left(10^{p_{10}}-1\right)\right\rceil$.

So, the proposed algorithm works for both conversion directions. However, one can notice, that for binary-to-decimal conversion the table size can be even reduced by the factor of two. We have used the mantissas from one binade: $2^{p_{2}-1} \leq m<2^{p_{2}}$. The whole reasoning stays the same if we scale these bounds in order to have $1 \leq m<2$, the table entries quantity stays the same. Now it is clear that $\left\lfloor\log _{10}(m)\right\rfloor=0$ for all these mantissas. However, it still stays zero if we slightly modify the mantissa's bounds: $\forall m^{\prime}: 1 \leq m^{\prime}<4, \log _{10}\left(m^{\prime}\right)=0$. Thus, we get a new binary representation of the input: $2^{E^{\prime}} m^{\prime}=2^{E} m$, where $E^{\prime}=E-(E \bmod 2)$ and $m^{\prime}=m \cdot 2^{E \bmod 2}$. So, we see that for the new mantissas interval we do not take into account the last exponent bit. So, the table entries quantity for the values $m^{*}(E)$ reduces twice. The corresponding interval for mantissas is $[1,4)$, because in this case we need to find the remainders of two, that is just a binary shift. The interval $[1,8)$ is larger, so it could reduce the table size even more, but requires computation of the remainders of three.

The table sizes for some particular formats are small enough to be integrated in hardware. However, these tables are quite multipurpose, they are shared between all I/O and arithmetic decimal-binary FP conversions, so, once they are coded, they could be used in all the mixed radix operations. The corresponding table sizes for different formats are listed in table I. 


\begin{tabular}{|c|l|}
\hline Initial Format & Table size \\
\hline binary32 & 554 bytes \\
\hline binary64 & 8392 bytes \\
\hline binary128 & 263024 bytes \\
\hline decimal32 & 792 bytes \\
\hline decimal64 & 6294 bytes \\
\hline decimal128 & 19713 bytes \\
\hline
\end{tabular}

Table I. TABLE SIZE FOR EXPONENT COMPUTATION STEP

\section{Computing the Mantissa With the Right ACCURACY}

As it was mentioned, the problem on the second step is the computation of the value $5^{B}$ with some bounded exponent $B \in \mathbb{N}$. If the initial range for the exponent of five contains negative values, we compute $5^{B+\bar{B}}$, where $\bar{B}$ is chosen in order to make the range for the exponents nonnegative. In this case we store the leading bits of $5^{-\bar{B}}$ as a constant and after computing $5^{B+\bar{B}}$ with the proposed algorithm, we multiply the result by the constant.

In this section we propose an algorithm for raising five to a huge natural power without rational arithmetic or divisions. The range for these natural exponents $B$ is determined by the input format, e.g. for the conversion from binary64 the range is about six hundred.

We propose to perform several Euclidean divisions in order to represent the number $B$ the following way:

$$
B=2^{n_{k}} \cdot q_{k}+2^{n_{k-1}} q_{k-1}+\ldots+2^{n_{1}} q_{1}+q_{0},
$$

where $0 \leq q_{0} \leq 2^{n_{1}}-1, n_{k} \geq n_{k-1}, k \geq 1$. The mentioned divisions are just a chain of binary shifts. All the quotients are in the same range and we assume that the range for $q_{0}$ is the largest one, so we have $q_{i} \in\left[0 ; 2^{n_{1}}-1\right], 0 \leq i \leq k$. Once the exponent is represented as (4), computation $5^{B}$ is done with the respect to the following expression:

$$
5^{B}=\left(5^{q_{k}}\right)^{2^{n_{k}}} \cdot\left(5^{q_{k-1}}\right)^{2^{n_{k-1}}} \cdot \ldots \cdot\left(5^{q_{1}}\right)^{2^{n_{1}}} \cdot 5^{q_{0}}
$$

Let us analyze how the proposed formula can simplify the algorithm of raising five to the power $B$. We mentioned that all the quotients $q_{i}$ are bounded. By selecting the parameters $k$ and $n_{i}$ we can make these quotients small, so the values $5^{q_{i}}$ can be stored in a table. Then, each factor in (5) is a table value raised to the power $2^{n_{i}}$ which is the same as a table value squared $n_{i}$ times.

So, the algorithm is the following: represent $B$ as (4) and get the values $q_{i}$, then for each $q_{i}$ get the table value $5^{q_{i}}$ and perform $n_{i}$ squarings, and finally multiply all the squared values beginning from the largest one. The scheme can be found on Fig. 1, the pseudocode for squarings is in algorithm 2 and for the final multiplication step in algorithm 3. All these steps are done in order to convert the FP numbers, so we simulate usual floating-point computations in integer. The exponent $B$ is huge, the value $5^{B}$ is also huge, so we can store only the leading bits. Thus, on each multiplication step (squarings are also multiplications) we throw away the last $\lambda$ bits. Of course these manipulations yield to an error, in section $\mathrm{V}$ there are details and proofs for the error analysis.

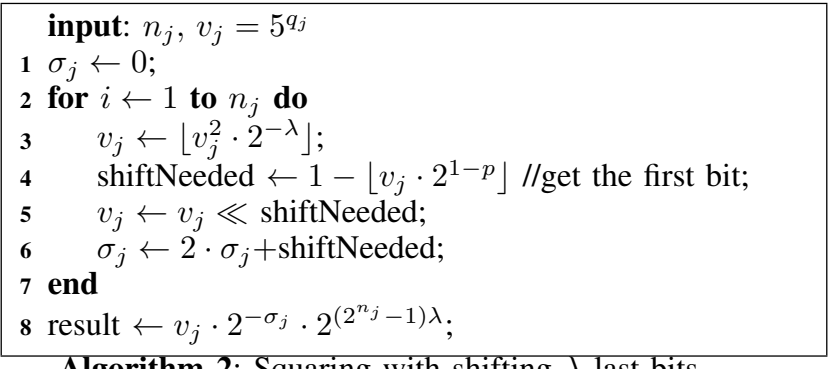

Algorithm 2: Squaring with shifting $\lambda$ last bits

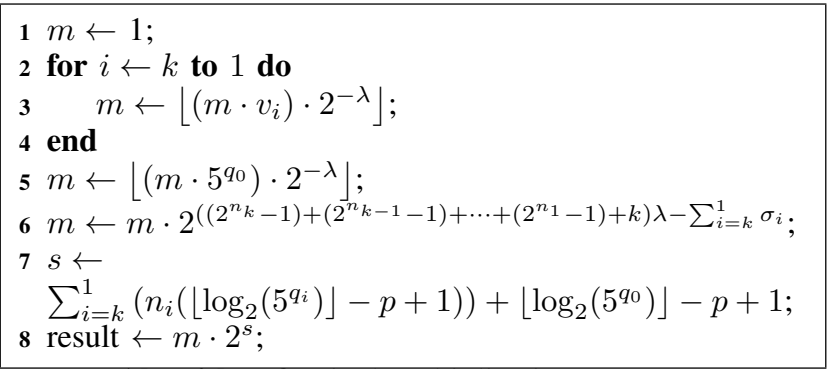

Algorithm 3: Final multiplication step

There is still one detail in algorithm 2 that was not explained: the correction $\sigma_{j}$. The mantissa of the input number is represented as a binary number bounded by one binade (for both, binary and decimal formats). Assume that we operate the numbers in the range $\left[2^{p-1}, 2^{p}\right)$. After each squaring we can get a value less then infimum of this range. So, if the first bit of the intermediate result after some squarings is 0 , we shift it to the left.

The described algorithm is applied $k$ times to each factors in (5). Then the last step is to multiply all the factors starting from the largest power like in listing below.

The whole algorithm schema is presented on Fig. 1. Depending on the range of $B$ one can represent it in different manner, but for our conversion tasks the ranges for $B$ were not that large, so the numbers $n_{j}$ were not more than 10 and the loops for squarings can be easily unrolled. For instance, for the conversions from binary32, binary64, decimal32 and decimal64 one can use the expansion of $B$ of the following form:

$$
B=2^{8} \cdot q_{2}+2^{4} \cdot q_{1}+q_{0}
$$

\section{ERROR ANALYSIS}

In order to compute the mantissa we use integer arithmetic but on each squaring/multiplication step we throw away a certain quantity of bits. So the final error is due to these right shiftings on each multiplication step.

We have errors only due to the multiplications, and as we do a lot of them, we need to define $N$ as the number of all the multiplications (squaring is just a particular case of multiplication). For each $i$-th factor $(1 \leq i \leq N)$ in (5) we need to perform $n_{i}$ squarings, thus it gives us $n_{i}$ multiplications. 


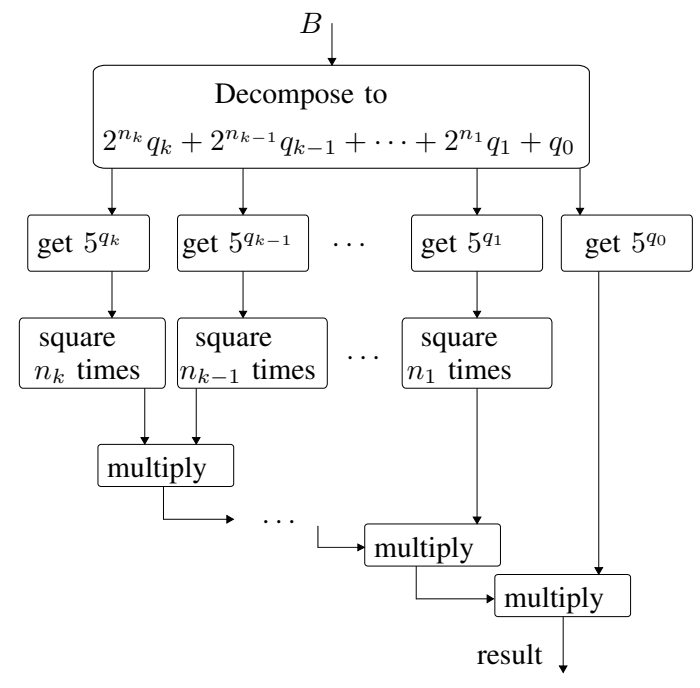

Figure 1. Raising 5 to a huge power

In order to get the final result we have to perform $k$ more multiplications, so the final expression for the $N$ constant is

$$
N=\sum_{i=1}^{k} n_{i}+k
$$

So, the result is a product of $N$ factors and on each step we have some relative error $\varepsilon_{i}$. This means, that if we define $y$ as the exact product without errors, then what we really compute in our algorithm can be represented as following:

$$
\hat{y}=y \prod_{i=1}^{N}\left(1+\varepsilon_{i}\right)
$$

Thus, the relative error of the computations is

$$
\varepsilon=\frac{\hat{y}}{y}-1=\prod_{i=1}^{N}\left(1+\varepsilon_{i}\right)-1
$$

Let us prove a lemma that will help us to find the bounds for the relative error of the result.

Lemma 1. Let $N \geq 3,0 \leq \bar{\varepsilon}<1$ and $\left|\varepsilon_{i}\right| \leq \bar{\varepsilon}$ for all $i \in[1, N]$. Then the following holds:

$$
\left|\prod_{i=1}^{N}\left(1+\varepsilon_{i}\right)-1\right| \leq(1+\bar{\varepsilon})^{N}-1
$$

Proof: This inequality is equivalent to the following:

$$
-(1+\bar{\varepsilon})^{N}+1 \leq \prod_{i=1}^{N}\left(1+\varepsilon_{i}\right)-1 \leq(1+\bar{\varepsilon})^{N}-1
$$

The proof of the right side is trivial. From the lemma 7 condition we have $-\bar{\varepsilon} \leq \varepsilon_{i} \leq \bar{\varepsilon}$, which is the same as 8 $1-\bar{\varepsilon} \leq \varepsilon_{i}+1 \leq \bar{\varepsilon}+1$ for arbitrary $i$ from the interval ${ }^{9}$ $[1, N]$. Taking into account the borders for $\bar{\varepsilon}$, we get that ${ }^{10}$ $0<\left(1+\varepsilon_{i}\right)<2$ for all $i \in[1, N]$. This means that we ${ }^{1}$ can multiply the inequalities $1+\varepsilon_{i} \leq \bar{\varepsilon}+1$ by $1+\varepsilon_{j}$ with $j \neq i$. After performing $N-1$ such multiplications and taking into account that $1+\varepsilon_{i} \leq \bar{\varepsilon}+1$, we get the following:

$$
\prod_{i=1}^{N}\left(\varepsilon_{i}+1\right) \leq(\bar{\varepsilon}+1)^{N} \text {. }
$$

So, the right side is proved.

The same reasoning applies for the left bounds from the lemma condition, and the family of inequalities $1-\bar{\varepsilon} \leq \varepsilon_{i}+1$ leads to the condition:

$$
(1-\bar{\varepsilon})^{N}-1 \leq \prod_{i=1}^{N}\left(1+\varepsilon_{i}\right)-1 .
$$

So, in order to prove the lemma we have to prove now that

$$
-(1+\bar{\varepsilon})^{N}+1 \leq(1-\bar{\varepsilon})^{N}-1 .
$$

After regrouping the summands we get the following expression to prove:

$$
2 \leq(1+\bar{\varepsilon})^{N}+(1-\bar{\varepsilon})^{N} .
$$

Using the binomial coefficients this trasforms to

$$
2 \leq 1+\sum_{i=1}^{N}\left(\begin{array}{c}
N \\
i
\end{array}\right) \bar{\varepsilon}^{i}+1+\sum_{i=1}^{N}\left(\begin{array}{c}
N \\
i
\end{array}\right)(-\bar{\varepsilon})^{i}
$$

On the right side of this inequality we always have the sum of 2 and some nonnegative terms. So, the lemma is proven.

The error $\bar{\varepsilon}$ is determined by the basic multiplication algorithm. It takes two input numbers (each of them is bounded between $2^{p-1}$ and $2^{p}$ ), multiplies them and cuts $\lambda$ last bits, see line 3 of algorithms 2 and 3. Thus, instead of $v_{j}^{2}$ on each step we get $v_{j}^{2} 2^{-\lambda}+\delta$, where $-1<\delta \leq 0$. So, the relative error of the multiplication is bounded by $|\bar{\varepsilon}| \leq 2^{-2 p+2+\lambda}$.

\section{IMPLEMENTATION DETAILS}

While the implementation of the first step is relatively simple, we need to specify some parameters and techniques that we used to implement raising 5 to an integer power.

The used computational precision $p$ was equal to 128 bits. The standard $\mathrm{C}$ integer types give us either 32 or 64 bits, so for the implementation we used the uint128_t type from GCC that is realised with two 64-bit numbers. As a shifting parameter $\lambda$ we took 64, so getting most or least 64 bits out of uint128_t number is easy and fast. Squarings and multiplications can be easily implemented using typecastings and appropriate shifts. Here, for instance, we put the code of squaring the 64-bit integer. The function returns two 64-bit integers, so the high and the low word of the 128-bit number.

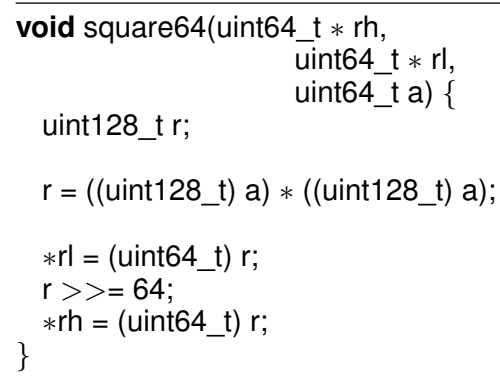

Listing 1. Example. C code sample for squaring a 64-bit number. 


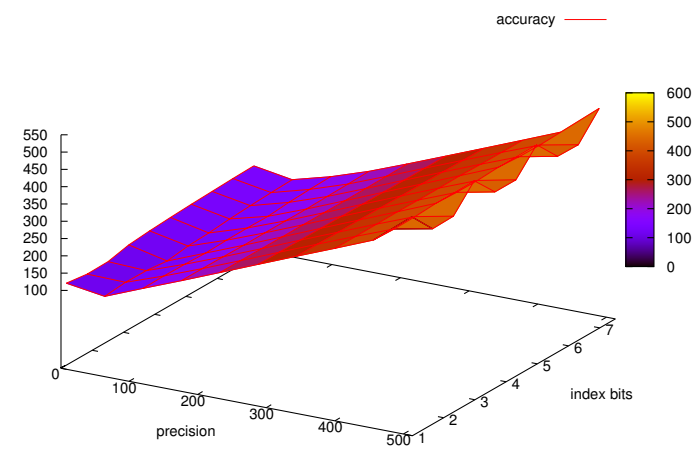

Figure 2. Accuracy as a function of precision and table index size

The other functions were implemented in the same manner.

We have implemented an run parametrized algorithm for computation of $5^{B}$, as the parameter we took the table index size (for entries $5^{q_{i}}$ ) and the working precision $p$. We see (Fig. 2) that the accuracy depends almost linearly on the precision.

\section{CONCLUSIONS}

A novel algorithm for conversion between binary and decimal floating-point representations has been presented. All the computations are done in integer arithmetic, so no FP flags or modes can be influenced. This means that the corresponding code can be made reentrant. The exponent determination is exact and can be done with several basic arithmetic operations, stored constants and a table. The mantissa computation algorithm uses a small exact table. The error analysis is given and it corresponds to the experimental results. The accuracy of the result depends on the computing precision and the table size. The conversions are often used and the tables are multipurpose, so they can be reused by dozens of algorithms. As this conversion scheme is used everywhere and the tables are not large, they might be integrated in hardware. The implementation of the proposed algorithm can be done without loops, so it reduces the instructions that control the loop, optimizes and therefore accelerates the code. The described conversion approach was used in the implementation of the scanf analogue in libieee 754 library [10].

\section{REFERENCES}

[1] IEEE Computer Society, IEEE Standard for Floating-Point Arithmetic. IEEE Standard 754-2008, Aug. 2008.

[2] J. Harrison, "Decimal transcendentals via binary," in Proceedings of the 2009 19th IEEE Symposium on Computer Arithmetic, ser. ARITH '09. Washington, DC, USA: IEEE Computer Society, 2009, pp. 187-194.

[3] M. Cornea, J. Harrison, C. Anderson, P. T. P. Tang, E. Schneider, and E. Gvozdev, "A Software Implementation of the IEEE 754R Decimal Floating-Point Arithmetic Using the Binary Encoding Format," IEEE Transactions on Computers, vol. 58, no. 2, pp. 148-162, 2009.
[4] N. Brisebarre, C. Lauter, M. Mezzarobba, and J.-M. Muller, "Comparison between binary64 and decimal64 floating-point numbers," in Proceedings of the 2013 IEEE 21st Symposium on Computer Arithmetic, April 2013.

[5] J.-M. Muller, N. Brisebarre, F. de Dinechin, C.-P. Jeannerod, V. Lefèvre, G. Melquiond, N. Revol, D. Stehlé, and S. Torres, Handbook of Floating-Point Arithmetic. Boston: Birkhäuser, 2010.

[6] D. M. Gay, "Correctly rounded binary-decimal and decimal-binary conversions," Numerical Analysis Manuscript 90-10, ATT Bell Laboratories, Tech. Rep., 1990.

[7] G. L. Steele and J. L. White, "How to print floating-point numbers accurately," SIGPLAN Not., vol. 39, no. 4, pp. 372-389, Jun. 1990.

[8] R. Burger and R. K. Dybvig, "Printing floating-point numbers quickly and accurately," in In Proc. of the ACM SIGPLAN '96 Conference on Programming Language Design and Implementation, 1996, pp. 108116.

[9] N. Brisebarre and J.-M. Muller, "Correctly rounded multiplication by arbitrary precision constants," IEEE Transactions on Computers, vol. 57, no. 2, pp. 165-174, Feb. 2008.

[10] O. Kupriianova and C. Lauter, "The libieee754 compliance library for the IEEE754-2008 Standard," in 15th GAMM - IMACS International Symposion on Scientific Computing, Computer Arithmetic and Validated Numerics, September 2012. 